ANNALES

POLONICI MATHEMATICI

$87(2005)$

\title{
Recent progress on the Jacobian Conjecture
}

\author{
by Michiel De Bondt and Arno van Den Essen (Nijmegen)
}

In memory of S. Lojasiewicz

\begin{abstract}
We describe some recent developments concerning the Jacobian Conjecture (JC). First we describe Drużkowski's result in [6] which asserts that it suffices to study the JC for Drużkowski mappings of the form $x+(A x)^{* 3}$ with $A^{2}=0$. Then we describe the authors' result of [2] which asserts that it suffices to study the JC for so-called gradient mappings, i.e. mappings of the form $x-\nabla f$, with $f \in k^{[n]}$ homogeneous of degree 4 . Using this result we explain Zhao's reformulation of the JC which asserts the following: for every homogeneous polynomial $f \in k^{[n]}$ (of degree 4) the hypothesis $\Delta^{m}\left(f^{m}\right)=0$ for all $m \geq 1$ implies that $\Delta^{m-1}\left(f^{m}\right)=0$ for all large $m$ ( $\Delta$ is the Laplace operator). In the last section we describe Kumar's formulation of the JC in terms of smoothness of a certain family of hypersurfaces.
\end{abstract}

Introduction. Since the first appearance of the JC in [12] various papers have been published concerning this conjecture. One of the milestones is undoubtedly the classical 1982 paper [1] of Bass, Connell and Wright. This paper gave an impulse to the field of polynomial automorphisms, which is now flourishing as never before. To mention a few highlights: the counterexample to the real Jacobian Conjecture by Pinchuk in [14], 1994, proofs of the 2-dimensional Markus-Yamabe Conjecture by Glutsuk, Fessler and Gutierrez in [9], [8] and [10], the polynomial counterexamples to the MarkusYamabe Conjecture in all dimensions $\geq 3$ by Cima, van den Essen, Gasull, Hubbers and Mañosas in [4], 1995, the proof of the linearization conjecture for $\mathbb{C}^{*}$-actions on $\mathbb{C}^{3}$ by Kaliman, Koras, Makar-Limanov and Russell in [11] and recently the negative solution of the tame generators conjecture by Shestakov and Umirbaev in [15]. However, since the famous reduction theorems of Bass, Connell, Wright/Yagzhev [16] and Drużkowski [5], not much progress has been made towards the Jacobian Conjecture.

The aim of this paper is to report on some surprising new reduction theorems, which go far beyond the classical reductions mentioned before. The

2000 Mathematics Subject Classification: Primary 14R15.

Key words and phrases: Jacobian Conjecture, Hessian Conjecture, Laplace operator. 
two most important papers in this respect are [2] and [17]. In the authors' paper [3] a survey is given of various results related to the paper [2]. Therefore in this paper we will focus our attention on Zhao's paper [17] (see Section 3). First we recall in Section 2 the main result of [2], on which Zhao's result is based. Finally, in the last section we describe one more consequence of the main theorem of [2]: namely a reformulation of the JC, due to Mohan Kumar, in terms of smoothness of a family of hypersurfaces.

\section{The classical reduction theorems and Drużkowski's recent re-} duction. Throughout this paper $k$ denotes an algebraically closed field of characteristic zero, and by $k^{[n]}$ or $k[x]$ we denote the $n$-variable polynomial ring $k\left[x_{1}, \ldots, x_{n}\right]$. Recall that the Jacobian Conjecture asserts that a polynomial map $F: k^{n} \rightarrow k^{n}$ is invertible if $\operatorname{det} J F \in k^{*}$, where $J F=\left(\partial F_{i} / \partial x_{j}\right)$ denotes the Jacobian matrix of $F$.

In [1] Bass, Connell and Wright and in [16] Yagzhev showed that it suffices to investigate the JC for all $n \geq 1$ and all polynomial maps of the form $F=x+H$, where $H=\left(H_{1}, \ldots, H_{n}\right)$ is homogeneous (of degree 3 ) and $J H$ nilpotent (in fact they show that for such homogeneous maps $H$ the condition $\operatorname{det} J F \in k^{*}$ is equivalent to $J H$ being nilpotent). A little later Drużkowski in [5] showed that one may even assume that each $H_{i}$ is of the form $L_{i}^{3}$, where $L_{i}$ is a linear form. In other words it suffices to study the JC for polynomial maps of the form $x+(A x)^{* 3}$, where $A \in M_{n}(k)$ and $\left(v_{1}, \ldots, v_{n}\right)^{* 3}$ denotes the vector $\left(v_{1}^{3}, \ldots, v_{n}^{3}\right)$. More recently Drużkowski in [6] obtained the following improvement of his reduction theorem.

THEOREM 1.1 (Drużkowski, 2000). It suffices to investigate the JC for all $n \geq 1$ and all polynomial maps of the form $x+(A x)^{* 3}$ with the additional property that $A^{2}=0$.

Proof. Let

$$
F:=x+(A x)^{* 3}: k^{n} \rightarrow k^{n}
$$

and let $i \in k$ satisfy $i^{2}=-1$. Put $F_{*}:=x+2 i(A x)^{* 3}$. Observe that $F_{*}=$ $z F\left(z^{-1} x\right)$, where $z^{2}=1 / 2 i$. So $F$ is invertible iff $F_{*}$ is invertible iff

$$
\widehat{F}:=\left(F_{*}, y\right)=\left(x+2 i(A x)^{* 3}, y\right): k^{2 n} \rightarrow k^{2 n}
$$

is invertible. Now put

$$
Q:=\left(x+i y, y+(A(x+i y))^{* 3}\right) \quad \text { and } \quad S:=(x-i y, y) .
$$

Then $G:=S \circ \widehat{F} \circ Q$ is invertible iff $\widehat{F}$ is invertible. Furthermore, one readily verifies that $G=(x, y)+(N(x, y))^{* 3}$, where

$$
N:=\left(\begin{array}{cc}
-i A & A \\
A & i A
\end{array}\right)
$$

satisfies $N^{2}=0$. 
2. Reduction to the symmetric case. Let $J H$ be a Jacobian matrix. Then one easily verifies that $J H$ is symmetric iff $H$ is a gradient mapping, i.e. $H=\nabla f\left(=\left(f_{x_{1}}, \ldots, f_{x_{n}}\right)\right)$ for some $f \in k[x]$. The main result of [2] asserts that it suffices to investigate the JC for all $n \geq 2$ and all $F: k^{n} \rightarrow k^{n}$ of the form $F=x+\nabla f$ (with $J(\nabla f)$ nilpotent). More precisely, we have

Theorem 2.1 (de Bondt and van den Essen, 2003). If the JC is true for all polynomial maps $F: k^{2 n} \rightarrow k^{2 n}$ of the form $x+\nabla f$, with $J(\nabla f)$ nilpotent (and homogeneous), then the JC is true for all polynomial maps of the form $x+H: k^{n} \rightarrow k^{n}$ with $J H$ nilpotent (and homogeneous).

The proof of this result is based on the next lemma. Recall that

$$
J(\nabla f)=\left(\frac{\partial^{2} f}{\partial x_{i} \partial x_{j}}\right)=: \mathcal{H}(f)
$$

is the Hessian of $f$. The standard bilinear form on $k^{n}$ is denoted by $\langle$,$\rangle .$

Lemma 2.2. Let $H=\left(H_{1}(x), \ldots, H_{n}(x)\right) \in k[x]^{n}$ and let $y_{1}, \ldots, y_{n}$ be new variables. Put

$$
f:=f_{H}=(-i)\langle H(x+i y), y\rangle .
$$

Then $J H$ is nilpotent iff $\mathcal{H}(f)$ is nilpotent.

Proof. (1) $\mathcal{H}(f)$ is nilpotent iff $\operatorname{det}\left(T I_{2 n}-\mathcal{H}(f)\right)=T^{2 n}$. Define $S:=$ $(x-i y, y)$ and let $S_{0}$ be the corresponding matrix in $M_{2 n}(k)$. Then $g:=$ $f \circ S=(-i)\langle H(x), y\rangle$ and

$$
\mathcal{H}(g)=\left(\begin{array}{cc}
* & (-i)(J H)^{t} \\
(-i) J H & 0
\end{array}\right) .
$$

Furthermore

$$
\mathcal{H}(g)=S_{0}^{t} \mathcal{H}(f)_{\mid S(x, y)} S_{0} .
$$

Since det $S_{0}=1$ we infer from (1) and (3) that $\mathcal{H}(f)$ is nilpotent iff

$$
\operatorname{det} S_{0}^{t}\left(T I_{2 n}-\mathcal{H}(f)\right)_{\mid S(x, y)} S_{0}=T^{2 n} \quad \text { iff } \quad \operatorname{det}\left(T S_{0}^{t} S_{0}-\mathcal{H}(g)\right)=T^{2 n} .
$$

Since

$$
S_{0}^{t} S_{0}=\left(\begin{array}{cc}
I_{n} & -i I_{n} \\
-i I_{n} & 0
\end{array}\right)
$$

we deduce from (2) that

$$
\mathcal{H}(f) \text { is nilpotent } \quad \text { iff } \quad \operatorname{det}\left(\begin{array}{cc}
* & -i T I_{n}+i(J H)^{t} \\
-i T I_{n}+i J H & 0
\end{array}\right)=T^{2 n} .
$$

Since for $n \times n$ matrices $A$ and $B$ we have

$$
\operatorname{det}\left(\begin{array}{cc}
* & -i A \\
-i B & 0
\end{array}\right)=\operatorname{det} A \operatorname{det} B
$$


we see that $\mathcal{H}(f)$ is nilpotent iff $\operatorname{det}\left(T I_{n}-(J H)^{t}\right) \operatorname{det}\left(T I_{n}-J H\right)=T^{2 n}$ iff $\operatorname{det}\left(T I_{n}-J H\right)=T^{n}$ iff $J H$ is nilpotent.

Proof of Theorem 2.1. Let $H=\left(H_{1}(x), \ldots, H_{n}(x)\right)$ with $J H$ nilpotent (and $H$ homogeneous). Let $f=f_{H}$ be as in Lemma 2.2. Then $\mathcal{H}(f)$ is nilpotent (and $f$ is homogeneous). So by our hypothesis

$$
G:=\left(x_{1}+f_{x_{1}}, \ldots, x_{n}+f_{x_{n}}, y_{1}+f_{y_{1}}, \ldots, y_{n}+f_{y_{n}}\right)
$$

is invertible. Consequently, with $S$ as in the proof of Lemma 2.2, $S^{-1} \circ G \circ S$ is also invertible. An easy calculation shows that

$$
S^{-1} \circ G \circ S=\left(x_{1}+H_{1}(x), \ldots, x_{n}+H_{n}(x), *, \ldots, *\right) .
$$

Since this last map is invertible, the desired result follows from the next lemma.

LeMma 2.3. If $\widetilde{F}:=\left(F_{1}(x), \ldots, F_{n}(x), *, \ldots, *\right): k^{2 n} \rightarrow k^{2 n}$ is invertible, then $F:=\left(F_{1}(x), \ldots, F_{n}(x)\right): k^{n} \rightarrow k^{n}$ is invertible.

Proof. Let $\left(G_{1}(x, y), \ldots, G_{n}(x, y), *, \ldots, *\right)$ be the inverse of $\widetilde{F}$. Then in particular

$$
F_{i}\left(G_{1}(x, y), \ldots, G_{n}(x, y)\right)=x_{i} \quad \text { for all } i .
$$

So $F_{i}\left(G_{1}(x, 0), \ldots, G_{n}(x, 0)\right)=x_{i}$ for all $i$, which means that $F$ is invertible with inverse $\left(G_{1}(x, 0), \ldots, G_{n}(x, 0)\right)$.

Combining Theorem 2.1 with the classical Bass-Connell-Wright/Yagzhev reduction theorem we get

COROLlary 2.4. The following statements are equivalent:

(i) The Jacobian Conjecture.

(ii) The Jacobian Conjecture for polynomial maps of the form $x+\nabla f$ with $\mathcal{H}(f)$ nilpotent and $f$ homogeneous of degree 4.

\section{Zhao's Laplace operator formulation of the Jacobian Conjec-} ture. In the previous section we saw that it suffices to investigate the JC for polynomial maps of the form $x+\nabla f$ with $\mathcal{H}(f)(=J(\nabla f))$ nilpotent (and we may even assume that $f$ is homogeneous of degree 4 ).

In [17] Zhao uses this result to obtain a remarkable reformulation of the JC. Recall that the Laplace operator, denoted $\Delta$, is equal to $\partial_{1}^{2}+\cdots+\partial_{n}^{2}$ $\left(\partial_{i}:=\partial / \partial x_{i}\right)$.

TheOREM 3.1 (Zhao, 2004). The JC is equivalent to each of the following statements.

(i) If $f$ is a homogeneous polynomial of degree $\geq 3$ such that $\Delta^{m}\left(f^{m}\right)=0$ for all $m \geq 1$, then $\Delta^{m-1}\left(f^{m}\right)=0$ for all large $m$.

(ii) If $f$ is a homogeneous polynomial of degree 4 such that $\Delta^{m}\left(f^{m}\right)=0$ for all $m \geq 1$, then $\Delta^{m-1}\left(f^{m}\right)=0$ for all large $m$. 
In the remainder of this section we give a somewhat simplified proof of this result. We start with some notations and generalities.

If $R$ is a commutative ring, then $R[[x]]$ denotes the ring $R\left[\left[x_{1}, \ldots, x_{n}\right]\right]$ of formal power series in $x_{1}, \ldots, x_{n}$ over $R$. The order of an element $g$ of $R[[x]]$, denoted $o(g)$, is by definition the smallest degree of a monomial appearing in $g$ if $g \neq 0$, and $o(g)=\infty$ if $g=0$. More generally, if $H=\left(H_{1}, \ldots, H_{n}\right) \in$ $R[[x]]^{n}$ then $o(H)$ denotes the minimum of the $o\left(H_{i}\right)$.

Now let $H \in k[[x]]^{n}$ with $o(H) \geq 2$. Then the formal map $F=x-H$ satisfies $\operatorname{det} J F(0)=1$. So it has a formal inverse. To study this inverse the crucial idea in [17] is to embed $F$ in a family of such maps. More precisely, let $t$ be a new variable and let $A:=k[t]$. Then define

$$
F_{t}:=x-t H(x) \in A[[x]]^{n} .
$$

Since $\operatorname{det}\left(J_{x} F_{t}\right)(0)=1$ it follows from the formal inverse function theorem $([7,1.1 .2])$ that $F_{t}$ has a unique formal inverse, say $G_{t}$ in $A[[x]]^{n}$, which is of the form $x+U_{t}(x)$ with $o\left(U_{t}\right) \geq 2$. Setting $t=0$ in $F_{t}\left(G_{t}(x)\right)=x$ we get $G_{0}(x)=x$. So $U_{t}(x)=t N_{t}(x)$ for some $N_{t}(x) \in A[[x]]^{n}$. Hence

$$
G_{t}(x)=x+t N_{t}(x) \text {. }
$$

Consequently, the equation $G_{t}\left(F_{t}(x)\right)=x$ implies that

$$
x-t H(x)+t N_{t}\left(F_{t}(x)\right)=x,
$$

whence

$$
N_{t}\left(F_{t}(x)\right)=H(x) .
$$

By the chain rule we get $J N_{t}\left(F_{t}\right) \cdot J F_{t}=J H$. Using $J F_{t}=I-t J H$ this gives

$$
J N_{t}\left(F_{t}\right)=J H \cdot(I-J H)^{-1}=\sum_{k=1}^{\infty}(J H)^{k}(x) t^{k-1} .
$$

Writing $\partial_{t}$ for $\partial / \partial t$ we get

Proposition 3.2. $N_{t}(x)$ is the unique formal solution of the Cauchy problem

$$
\partial_{t}\left(N_{t}\right)=J N_{t} \cdot N_{t}, \quad N_{t=0}(x)=H(x) .
$$

Proof. The initial condition follows directly from (4). Furthermore, differentiating (4) with respect to $t$ gives $\partial_{t}\left(N_{t}\right)\left(F_{t}\right)-\left(J N_{t}\right) H=0$. Composing from the right with $G_{t}$ and using (4) gives the desired result.

From now on we assume that $J H$ is symmetric. So $H=\nabla f$ for some unique $f \in k[[x]]$ with $o(f) \geq 3$. It follows from (5) that $J N_{t}\left(F_{t}\right)$ is symmetric and hence so is $J N_{t}(x)$. Consequently, there exists a unique $Q_{t} \in A[[x]]$ with $o\left(Q_{t}\right) \geq 3$ such that $N_{t}(x)=\nabla Q_{t}$. So $G_{t}(x)=x+t \nabla Q_{t}$. Writing $\langle$,$\rangle for$ the standard bilinear form we have 
Proposition 3.3. $Q_{t}$ is the unique solution of the Cauchy problem

$$
\partial_{t}\left(Q_{t}\right)=\frac{1}{2}\left\langle\nabla Q_{t}, \nabla Q_{t}\right\rangle, \quad Q_{t=0}=f .
$$

Proof. Using $N_{t}=\nabla Q_{t}$ and 3.2 we get

$$
\nabla\left(\partial_{t}\left(Q_{t}\right)\right)=\partial_{t}\left(\nabla Q_{t}\right)=J N_{t} \cdot \nabla Q_{t}
$$

Also one easily verifies that

$$
\nabla\left(\frac{1}{2}\left\langle\nabla Q_{t}, \nabla Q_{t}\right\rangle\right)=\mathcal{H}\left(Q_{t}\right) \cdot \nabla Q_{t}=J N_{t} \cdot \nabla Q_{t} .
$$

So $\nabla\left(\partial_{t}\left(Q_{t}\right)\right)=\nabla\left(\frac{1}{2}\left\langle\nabla Q_{t}, \nabla Q_{t}\right\rangle\right)$. This implies the first equality in (6), since the polynomials in this equation have no constant term. Finally, using (4) we find that $\nabla Q_{t=0}=N_{0}=H=\nabla f$, which gives $Q_{t=0}=f$.

In order to investigate JC one should, according to 3.4 study polynomial maps $x-\nabla f$ with $\mathcal{H}(f)$ nilpotent. Therefore we call an element $f \in k[[x]]$ for which the matrix $\mathcal{H}(f)$ is nilpotent, Hesse nilpotent, HN for short.

Lemma 3.4. Let $f \in k[[x]]$ with $o(f) \geq 3$. Then $f$ is $H N$ iff $\Delta Q_{t}=0$.

Proof. Observe that $J N_{t}=J\left(\nabla Q_{t}\right)=\mathcal{H}\left(Q_{t}\right)$, whence

$$
\operatorname{Tr} J N_{t}=\operatorname{Tr} \mathcal{H}\left(Q_{t}\right)=\Delta Q_{t} .
$$

Since $H=\nabla f$ we also have $J H=\mathcal{H}(f)$. Then it follows from (5) by taking traces that

$$
\left(\Delta Q_{t}\right)\left(F_{t}\right)=\sum_{k=1}^{\infty} \operatorname{Tr} \mathcal{H}(f)^{k} t^{k-1} .
$$

Finally, $f$ is $\mathrm{HN}$ iff $\operatorname{Tr} \mathcal{H}(f)^{k}=0$ for all $k \geq 1$ iff $\left(\Delta Q_{t}\right)\left(F_{t}\right)=0$ iff $\Delta Q_{t}=0$.

Now we are able to give Zhao's main theorem, which gives a beautiful formula for $Q_{t}$ (and hence for the formal inverse $G_{t}=x+\nabla Q_{t}$ ) in case $f$ is HN. In fact his theorem gives the following more general result.

Theorem 3.5 (Zhao, 2004). Let $f \in k[[x]]^{n}$ with $o(f) \geq 3$ and HN. Then

$$
Q_{t}^{k}=k ! \sum_{m=0}^{\infty} \frac{t^{m}}{2^{m} m !(m+k) !} \Delta^{m}\left(f^{m+k}\right) \quad \text { for all } k \geq 1 .
$$

Proof. Introduce a new variable $s$ and consider the generating function of the sequence $\left\{Q_{t}^{k} / k !\right\}$, i.e. $U:=\exp \left(s Q_{t}\right)$.

Claim. $U$ is the unique solution of the Cauchy problem

$$
\partial_{t}(U)=\frac{1}{2 s} \Delta U, \quad U(t=0)=\exp (s f) .
$$

To prove this claim observe that, using (6), we get

$$
\partial_{t}(U)=\frac{s}{2}\left\langle\nabla Q_{t}, \nabla Q_{t}\right\rangle U \quad \text { and } \quad U(t=0)=\exp (s f) .
$$


Furthermore,

$$
\begin{aligned}
\Delta U & =s \sum_{i} \partial_{i}\left(\partial_{i}\left(Q_{t}\right) U\right)=s \Delta\left(Q_{t}\right) U+s^{2} \sum_{i} \partial_{i}\left(Q_{t}\right)^{2} U \\
& =s^{2}\left\langle\nabla Q_{t}, \nabla Q_{t}\right\rangle U
\end{aligned}
$$

since $\Delta Q_{t}=0$ by 3.4. From (10) and (11) we get (9). However, also the formal series

$$
\sum_{k=0}^{\infty} \frac{t^{k}}{(2 s)^{k} k !} \Delta^{k}(\exp (s f))
$$

is a solution of the Cauchy problem (9), as one easily verifies. So by uniqueness this series is equal to $\exp \left(s Q_{t}\right)$. Comparing the coefficients of $s^{k}$ for all $k \geq 1$ we obtain (8).

As an immediate consequence of (8) we get

Corollary 3.6. Let $f \in k[[x]]$ with $o(f) \geq 3$ and $f H N$. Then $\Delta^{m}\left(f^{m}\right)$ $=0$ for all $m \geq 1$.

Proof. By 3.4, $\Delta Q_{t}=0$. Then use (8) with $k=1$.

Now we show that the converse holds as well:

THEOREM 3.7. Let $f \in k[[x]]$ with $o(f) \geq 3$. Then $f$ is $H N$ iff $\Delta^{m}\left(f^{m}\right)$ $=0$ for all $m \geq 1$ iff $\Delta^{m}\left(f^{m}\right)=0$ for all $1 \leq k \leq n$.

This follows directly from the next result with $k=n$, using the fact that an $n \times n$ matrix $A$ over a domain is nilpotent iff $\operatorname{Tr} A^{k}=0$ for all $1 \leq k \leq n$.

Proposition 3.8. Let $v_{m}(f):=\Delta^{m}\left(f^{m}\right)$ and $u_{m}(f):=\operatorname{Tr} \mathcal{H}(f)^{m}$ for all $m \geq 1$. Let $k \geq 1$. If $v_{1}(f)=\cdots=v_{k}(f)=0$, then $u_{1}(f)=\cdots=u_{k}(f)=0$.

The proof of this result is based on the following lemma in which we use the symbol "*" to denote a non-zero constant in $k$.

LEMMA 3.9. Let $k \geq 1$ and $u_{1}(f)=\cdots=u_{k}(f)=0$. Then for all $m \geq 1$,

$$
\partial_{t}^{l} Q_{t}^{m} \equiv * \Delta^{l} Q_{t}^{m+l}\left(\bmod t^{(k+1)-l}\right) \quad \text { for all } 1 \leq l \leq k .
$$

Proof. By induction on $l$. For the case $l=1$, observe that

$$
\partial_{t} Q_{t}^{m}=m Q_{t}^{m-1} \partial_{t}\left(Q_{t}\right)=* Q_{t}^{m-1}\left\langle\nabla Q_{t}, \nabla Q_{t}\right\rangle \quad(\text { by }(6)) .
$$

So we need to show that $Q_{t}^{m-1}\left\langle\nabla Q_{t}, \nabla Q_{t}\right\rangle \equiv \Delta\left(Q_{t}^{m+1}\right)\left(\bmod t^{k}\right)$. To see this observe that

$$
\Delta\left(Q_{t}^{m+1}\right)=* Q_{t}^{m} \Delta Q_{t}+* Q_{t}^{m-1}\left\langle\nabla Q_{t}, \nabla Q_{t}\right\rangle
$$

Since by $(7)$ and the hypothesis $\Delta Q_{t} \equiv 0\left(\bmod t^{k}\right)$, the case $l=1$ follows.

Now assume (12) holds for some $l$ with $1 \leq l \leq k$. Applying $\partial_{t}$ to (12) gives

$$
\partial_{t}^{l+1} Q_{t}^{m} \equiv * \Delta^{l} \partial_{t}\left(Q_{t}^{m+l}\right)\left(\bmod t^{(k+1)-(l+1)}\right)
$$


From the case $l=1$ with $m+l$ instead of $m$ we get

$$
\partial_{t}\left(Q_{t}^{m+l}\right) \equiv * \Delta Q_{t}^{m+l+1}\left(\bmod t^{k}\right) .
$$

Combining this with (13) gives the desired result for $l+1$.

Proof of 3.8. By induction on $k$. The case $k=1$ is obvious since $v_{1}(f)=$ $u_{1}(f)$. So assume 3.8 for $k \geq 1$ and let us prove it for $k+1$. So we assume that $v_{1}(f)=\cdots=v_{k+1}(f)=0$. In particular the induction hypothesis implies that $u_{1}(f)=\cdots=u_{k}(f)=0$. So by $(7)$,

$$
\Delta Q_{t} \equiv u_{k+1}(f) t^{k}\left(\bmod t^{k+1}\right) .
$$

Consequently,

$$
u_{k+1}(f)=\frac{1}{k !} \partial_{t}^{k}\left(\Delta Q_{t}\right)_{t=0}
$$

Furthermore, applying $\Delta$ to (12) with $l=k$ and $m=1$ we get

$$
\partial_{t}^{k}\left(\Delta Q_{t}\right) \equiv * \Delta^{k+1} Q_{t}^{k+1}(\bmod t) .
$$

So, using $Q_{0}=f$ (by (6)), we get

$$
u_{k+1}(f)=* \Delta^{k+1} Q_{0}^{k+1}=* \Delta^{k+1} f^{k+1}=v_{k+1}=0,
$$

as desired.

Now we are finally able to give

Proof of Theorem 3.1. Let $f$ be homogeneous of degree 4. Substituting $t=1$ in (8) with $k=1$ we see that the formal inverse of $x-\nabla f$ is of the form $x+\nabla Q$, where

$$
Q=\sum_{m=0}^{\infty} \frac{1}{2^{m} m !(m+1) !} \Delta^{m}\left(f^{m+1}\right) .
$$

Since $\mathcal{H}(f)$ being nilpotent is equivalent to the conditions described in 3.7, the desired result follows readily from 2.4 .

4. Kumar's formulation of the Jacobian Conjecture. We conclude this paper with an observation of Mohan Kumar ([13]) which describes the Jacobian Conjecture as a problem concerning the smoothness of some hypersurfaces.

Theorem 4.1 (Kumar, 2004). The Jacobian Conjecture is equivalent to the following statement:

(S) For every homogeneous $H N$ polynomial $f$ of degree 4 , every $1 \leq i \leq n$ and every $t \in k^{*}$, the hypersurface $S(t, i)=0$, where

$$
S(t, i):=x_{i}+f_{x_{i}}+\frac{t}{2} f_{x_{i} x_{i}}+\frac{t^{2}}{6} f_{x_{i} x_{i} x_{i}},
$$

has no singularities. 
Proof. (i) First assume (S). Let $f$ be a homogeneous HN polynomial of degree 4. According to 2.4 and $[7,4.2 .1]$ it suffices to show that $F:=x+\nabla f$ is injective. Therefore suppose that $F(a)=F(a+b)$ for some $a, b \in k^{n}$ with $b \neq 0$. Choose an orthogonal matrix $T$ such that $T^{-1} b=(t, 0, \ldots, 0)$ for some $t \in k^{*}$. Put $g:=f \circ T$. Then $G:=x+\nabla g=x+T^{t} \circ \nabla f \circ T=T^{-1} \circ F \circ T$ and $G\left(T^{-1} a\right)=G\left(T^{-1} a+T^{-1} b\right)$. So replacing $F$ by $G$ and $f$ by $g$ we may assume that $b=(t, 0, \ldots, 0)$ for some $t \in k^{*}$.

(ii) Now consider the assumption

$$
(x+\nabla f)(a+(t, 0, \ldots, 0))=(x+\nabla f)(a) .
$$

Put $a_{*}:=\left(a_{2}, \ldots, a_{n}\right)$. Then looking at the first component of (14) we get $a_{1}+t+f_{x_{1}}\left(a_{1}+t, a_{*}\right)=a_{1}+f_{x_{1}}(a)$. Expanding $f_{x_{1}}\left(a_{1}+t, a_{*}\right)$ in its Taylor series we deduce that

$$
t+t f_{x_{1} x_{1}}(a)+\frac{t^{2}}{2} f_{x_{1} x_{1} x_{1}}(a)+\frac{t^{3}}{6} f_{x_{1} x_{1} x_{1} x_{1}}(a)=0 .
$$

For $2 \leq i \leq n$, looking at the $i$ th component of (14) gives

$$
t f_{x_{i} x_{1}}(a)+\frac{t^{2}}{2} f_{x_{i} x_{1} x_{1}}(a)+\frac{t^{3}}{6} f_{x_{i} x_{1} x_{1} x_{1}}(a)=0 .
$$

Dividing by $t \in k^{*}$ we deduce from (15) and (16) that the hypersurface $S(t, 1)=0$ has a singularity at $a$, contradiction.

(iii) Conversely, assume that the JC holds. If for some homogeneous HN polynomial $f$ of degree 4 , some $1 \leq i \leq n$ and some $t \in k^{*}$ the hypersurface $S(t, i)=0$ has a singularity, say at $a \in k^{n}$, then reading backwards the argument in (ii) we find from (14) that the map $x+\nabla f$ is not injective. In particular $x+\nabla f$ is not invertible, contradicting the $\mathrm{JC}$ since $f$ is HN, i.e. $J(\nabla f)$ is nilpotent.

To conclude this paper we give the following interesting observation, also due to Kumar.

Proposition 4.2. Let $f$ be a homogeneous $H N$ polynomial of degree 4 . Then for every $i \leq i \leq n$ and every $t \in k$ the hypersurface $R(t, i)=0$, where

$$
R(t, i):=x_{i}+f_{x_{i}}+t f_{x_{i} x_{i}}+\frac{t^{2}}{2} f_{x_{i} x_{i} x_{i}}
$$

has no singularities.

Proof. We may assume that $i=1$. Let $b:=(t, 0, \ldots, 0)$ and $x_{*}:=$ $\left(x_{2}, \ldots, x_{n}\right)$. Since $\mathcal{H}(f)$ is nilpotent, so is

$$
\mathcal{H}(f)\left(x_{1}+t, x_{*}\right)=\mathcal{H}\left(f\left(x_{1}+t, x_{*}\right)\right) .
$$

Using Taylor's expansion we get

$$
f\left(x_{1}+t, x_{*}\right)=f(x)+t f_{x_{1}}(x)+\frac{t^{2}}{2} f_{x_{1} x_{1}}(x)+\cdots .
$$


Since "taking the Hessian" of a polynomial is additive, we see that

$$
\mathcal{H}\left(f\left(x_{1}+t, x_{*}\right)\right)=\mathcal{H}(f)+t \mathcal{H}\left(f_{x_{1}}\right)+\frac{t^{2}}{2} \mathcal{H}\left(f_{x_{1} x_{1}}\right) .
$$

The first row of this matrix is $\nabla\left(R(t, 1)-x_{1}\right)$ and thus $\nabla(R(t, 1))$ is the first row of the invertible matrix $I_{n}-\mathcal{H}\left(f\left(x_{1}+t, x_{*}\right)\right)$, which implies that the hypersurface $R(t, 1)=0$ has no singularities.

Acknowledgements. The first author is sponsored by NWO, the Dutch Organisation for Scientific Research.

\section{References}

[1] H. Bass, E. Connell and D. Wright, The Jacobian Conjecture: Reduction of degree and formal expansion of the inverse, Bull. Amer. Math. Soc. 7 (1982), 287-330.

[2] M. de Bondt and A. van den Essen, A reduction of the Jacobian Conjecture to the symmetric case, Proc. Amer. Math. Soc. 133 (2005), 2201-2205.

[3] - - - Hesse and the Jacobian Conjecture, in: Contemp. Math. 369, Amer. Math. Soc., 2005, 63-76.

[4] A. Cima, A. van den Essen, A. Gasull, E. Hubbers and F. Mañosas, A polynomial counterexample to the Markus-Yamabe Conjecture, Adv. in Math. 131 (1997), 453457.

[5] L. Drużkowski, An effective approach to Keller's Jacobian Conjecture, Math. Ann. 264 (1983), 303-313.

[6] -, New reduction in the Jacobian Conjecture, in: Effective Methods in Algebraic and Analytic Geometry (Kraków, 2000), Univ. Iagel. Acta Math. 39 (2001), 203-206.

[7] A. van den Essen, Polynomial Automorphisms and the Jacobian Conjecture, Progr. Math. 190, Birkhäuser, 2000.

[8] R. Fessler, A solution of the two dimensional Global Asymptotic Jacobian Stability Conjecture, Ann. Polon. Math. 62 (1995), 45-75.

[9] A. Glutsuk, The complete solution of the Jacobian Problem for planar vector fields, Uspekhi Mat. Nauk 49 (1994), no. 3, 179-180 (in Russian).

[10] C. Gutierrez, A solution to the bidimensional Global Asymptotic Stability Conjecture, Ann. Inst. H. Poincaré Anal. Non Linéaire 12 (1995), 627-672.

[11] S. Kaliman, M. Koras, L. Makar-Limanov and P. Russell, $\mathbb{C}^{*}$-actions on $\mathbb{C}^{3}$ are linearizable, Electron. Res. Announc. Amer. Math. Soc. 3 (1997), 63-71.

[12] O. Keller, Ganze Cremona-Transformationen, Monatsh. Math. Phys. 47 (1939), 299-306.

[13] M. Kumar, private communication, May 19, 2004.

[14] S. Pinchuk, A counterexample to the real Jacobian Conjecture, Math. Z. 217 (1994), $1-4$.

[15] I. Shestakov and U. Umirbaev, The tame and wild automorphisms of polynomial rings in three variables, J. Amer. Math. Soc. 17 (2004), 197-227.

[16] A. Yagzhev, On Keller's problem, Siberian Math. J. 21 (1980), 747-754. 
[17] W. Zhao, Hessian nilpotent formal power series and their deformed inversion pairs, Trans. Amer. Math. Soc., to appear.

Department of Mathematics

University of Nijmegen

Postbus 9010

6500 GL Nijmegen, The Netherlands

E-mail: debondt@math.ru.nl

essen@math.ru.nl

Reçu par la Rédaction le 6.9.2004 